\title{
Freight Consolidation Problem with Time Windows, Pickup and Delivery Sequence
}

\author{
Devaraj R. Krishnan and Tieming Liu* \\ School of Industrial Engineering \& Management, Oklahoma State University, Stillwater, OK 74078
}

\begin{abstract}
The number of online market places for freight matching is on the rise. Online market places help small trucking companies find shipping customers. However, they do not provide cargo consolidation strategies. This lack of effective consolidation has adverse effects on the shipping industry and greenhouse gas emission. To that extent, this article addresses Multiple Vehicle Pickup and Delivery Problem with Time Windows (MVPDPTW). We propose a Mixed Integer Programming (MIP) model and a branch-andcut algorithm geared towards identifying effective freight consolidation opportunities. For emission studies, we used a cost conversion technique from the literature to convert emission levels into monetary values. On real-world logistics company test instances, our model identified routes with lower cost and lower emission levels than the actual routes.
\end{abstract}

\section{Introduction}

The trucking industry contributes more than $84 \%$ of revenue in U.S. commercial transportation sector. As a source of many direct and indirect employment opportunities, the trucking industry has big economic implications for the country.

However, the U.S. trucking industry is very fragmented. Currently there are over 110,000 carriers and 350,000 independent owner-operators [3]. Among them, around $97 \%$ of the carriers own less than 20 trucks and around $90 \%$ owns six or lesser trucks [1]. This fragmentation hinders the efficiency of cargo transportation. An estimated $15 \%$ to $25 \%$ of the trucks on road are travelling empty [2]. Furthermore, it is difficult for small shipping companies to find customers and fill full truckloads. This reduced efficiency causes hike in shipping prices, greenhouse gas emissions and traffic congestion. Further considering the unused spaces of non-empty trucks, there is a great need for better efficiency. Truck sharing is one such way to attain better efficiency in cargo transportation.

Internet and mobile computing technology have made truck sharing more viable. The number of online marketplaces for freight-matching is on the rise. The concept of freight-matching is like Uber which connects driver and passenger based on request. However, the working principle behind freight-equipment matching is more complicated than Uber, because of various sizes and types of freights and trucks. It is very difficult and time-consuming for carriers to search shippers' demand information online to identify freight consolidation opportunities. It will be very helpful if the online freightmatching marketplace could provide consolidation solutions to the carriers. Therefore, online market places are in great need for effective freight consolidation algorithms. Identifying effective consolidation techniques and quantifying the effect of consolidation on greenhouse gas emissions are the main objectives of our paper.

With the above motivation we study a Multiple Vehicles with Pickup and Delivery Problem with Time Windows (MVPDPTW) in this paper. By solving MVPDPTW, online marketplaces can identify effective consolidation strategies. This paper solves MVPDPTW with homogenous fleet (multiple vehicles with same capacity), pickup and delivery deadlines for each shipment order and maximum time a driver spends on road.

\section{Literature review}

Vehicle Routing Problem (VRP) with Pickup and Delivery is a well-studied problem in the literature. For general surveys on this problem, we refer the reader to Parragh et al. [1]. Hoff et al. [2] presented a comprehensive literature review to describe industrial aspects of combined fleet composition and routing. It classifies the problems in different categories: namely heterogeneous fleet problems, network design problems, fleet composition and routing problems. This taxonomy is a highly useful tool to understand and do further literature review on truck sharing.

Multiple Vehicle Pickup and Delivery Problem with Time Windows (MVPDPTW) is a category of VRP with a lot of practical implementations. Ropke et al. [3] presented two formulations and a column generation algorithm to solve one to one-pickup and delivery problem. The mathematical model used for this paper is very close to the first formulation presented by Ropke at al. [3]. However, we present a branch-and-cut algorithm

\footnotetext{
* Corresponding author: tieming.liu@okstate.edu
} 
which is very different from the column generation algorithm proposes in that paper.

Some literature has also focused on emission studies. Schulte et al., [4] presented a mathematical model to estimate gas emission and cost objective in transportation between urban ports and hinterland. Empty container transportation is a pervasive problem in ports. This paper presents an extensive study for truck routing models in a portside framework. However, this work presents a model for solving the problem on a restricted network structure. Hwang [5] presented techniques to relate freight movement with environmental impacts and traffic congestion. The emission and traffic congestion based dynamic routing approach presented in this thesis is a valuable application for urban transportation planning. The quantification methods for environmental impacts presented in this paper have been pivotal for emission studies in our project. The classification our literature review is as follows:

Table 1. Literature review categories.

\begin{tabular}{|c|c|}
\hline Category & Related literature \\
\hline VRP taxanomy & Parragh et al. [1], Hoff et al. [2] \\
\hline $\begin{array}{c}\text { Heterogenous fleet } \\
\text { VRP } \\
\text { Tavakkntilis and Kiranoudis [6], } \\
\text { Bettinelli et al [8] }\end{array}$ & $\begin{array}{c}\text { [7], } \\
\text { problem }\end{array}$ \\
\hline $\begin{array}{c}\text { Pickup and delivery } \\
\text { stronmental } \\
\text { studies }\end{array}$ & Ropke et al. [3], Haghani and Oh [9] \\
\hline
\end{tabular}

\section{Mathematical notations}

Let $\mathrm{n}$ denote the number of shipment requests. Consider a directed graph $G=(N, A)$ with node set $N=$ $\{0,1, \ldots, 2 n, 2 n+1\}$ and arc set $A$. Node subsets $\mathrm{P}=$ $\{1, \ldots, n\}$ and $D=\{n+1, \ldots, 2 n\}$ are pickup and delivery nodes respectively, whereas 0 and $2 n+1$ are origin and destination depots respectively. Each shipment order has an associated pickup node $i \in P$ and delivery node $\mathrm{n}+i \epsilon$ $D$. The arc set is given by $A=\{(0, i) \mid i \in P\} \mathrm{U}\{(n+i, 2 n$ +1) $i \in \in P\} \mathrm{U} A\left(K_{2 n}\right)$, where $A\left(K_{2 n}\right)$ is the arc set of complete graph on $P \mathrm{U} D$. Each directed are $(i, j) \in A$ has an associated cost value $c_{\mathrm{ij}}$. Each node $i \in N$ has an associated load value $q_{i}$, satisfying $q_{i}=-q_{n+i} \forall i \in P$ and $q_{i}$ $=0$ for $i=0,2 n+1$. Each node $i \in N$ also has an associated time window $\left[a_{i}, b_{i}\right]$, where $a_{i}$ and $b_{i}$ are the earliest and latest times at which service can start at node $i \in N$. For origin depot, $\mathrm{a}_{\mathrm{i}}$ and $\mathrm{b}_{\mathrm{i}}$ are the earliest and latest times are which vehicles may leave a depot respectively. The latest service time for the destination depot node represents the vehicle's maximum working hours.

Let $K$ denote a set of homogeneous vehicles with uniform capacity $Q$. The maximum number of vehicles available at the origin depot is $h$.

\section{Problem objectives}

Multiple Vehicles Pickup and Delivery Problem with Time Windows (MVPDPTW) is to identify the minimum cost routes while satisfying the following Conditions:

1. Each pickup or delivery node is visited exactly once by one vehicle.

2. The delivery node of each shipment is visited by same vehicle which visited the corresponding pickup node.

3. The number of routes must not exceed the number of available vehicles.

4. For each shipment and vehicle, the pickup node must be visited before the delivery node.

5. Each node can be visited only within the associated time window.

6. The cargo carried by each vehicle must not exceed the maximum weight capacity.

By solving the MVPDPTW, we can identify truck routes with optimal freight consolidation. Our objectives in this paper are:

1. To solve MVPDPTW for identifying effective truck consolidation routes.

2. To compare the environmental impact (greenhouse gas emission) of consolidated routes against actual routes used by a partnering shipping company.

\section{MIP formulation for MVPDPTW}

\subsection{Decision variables}

1. $x_{i j}^{k}=1$ if $(i, j) \in A$ is on the route of vehicle $k \epsilon$ $K ; x^{k}{ }_{i j}=0$ otherwise.

2. $Q^{k}{ }_{i}$ is the load on vehicle $k \in K$ upon leaving node $i \in N$.

3. $B^{k}{ }_{i}$ is the time by which vehicle $k \in K$ begins service at node $i \epsilon N$.

Formulation 1

$$
\begin{aligned}
& \operatorname{Min} \sum_{k \in K} \sum_{i \in N} \sum_{j \in N} c_{i j} x_{i j}^{k} \\
& \sum_{k \in K} \sum_{j \in N} x_{i j}^{k}=1 \quad \forall i \in P \\
& \sum_{j \in N} x_{i j}^{k}-\sum_{j \in N} x_{n+i, j}^{k}=0 \quad \forall i \in P, k \in K \\
& \sum_{j \in N} x_{0 j}^{k}=1 \quad \forall k \in K \\
& \sum_{j \in N} x_{i j}^{k}-\sum_{j \in N} x_{j i}^{k}=0 \quad \forall i \in P \cup D, k \in K \\
& Q_{j}^{k} \geq\left(Q_{i}^{k}+q_{j}\right) x_{i j}^{k} \quad \forall i, j \in N, \forall k \in K \\
& B_{j}^{k} \geq\left(B_{i}^{k}+t_{i j}\right) x_{i j}^{k} \quad \forall i, j \in N, \forall k \in K \\
& B_{i}^{k}+t_{i, n+i} \leq B_{n+i}^{k} \quad \forall i \in P, \forall k \in K \\
& a_{i} \leq B_{i}^{k} \leq b_{i} \quad \forall i \in N, \forall k \in K \\
& \max \left\{0, q_{i}\right\} \leq Q_{i}^{k} \leq \min \left\{Q, Q+q_{i}\right\} \quad \forall i \in N, \forall k \in K \\
& \sum_{i, j \in N, i \neq j} x_{i j}^{k} \leq|S|-1 \quad \forall k \in K, \forall S \subset N,|S| \geq 2 \\
& y_{k} \in\{0,1\} \quad \forall k \in K \\
& x_{i j}^{k} \in\{0,1\} \quad \forall i, j \in N, \forall k \in K
\end{aligned}
$$


The objective function seeks to minimize the total transportation cost. For the reader's convenience, we have explained the Conditions (from the above PROBLEM OBJECTIVES section) satisfied by each constraint in our model

- Constraints 2 and 5 are for Condition 1.

- Constraint 3 is for Condition 2.

- Constraint 4 is for Condition 3.

- Constraint 8 is the precedence constraint. It is for Condition 4.

- $\quad$ Constraints 7 and 9 are for Condition 5.

- Constraints 6 and 10 are for Condition 6 .

- Constraint 11 is the Sub-tour Elimination Constraints (SECs).

Note that Constraints 6 and 7 are non-linear. However, we can linearize the product of two variables using some standard linearization techniques.

\subsection{Branch-and-cut algorithm}

In this section, we discuss our approach to solve MVPDPTW. Constraints 11 in Formulation 1 is the well-known Dantzig-Fulkerson-Johnson (DFJ) sub-tour elimination constraint. For a non-trivial input graph, the number of DFJ constraints is exponential, which makes this problem hard to solve. Computationally, MVPDPTW is a NP-hard problem. So, using the traditional branch-and-bound algorithm to solve this problem might not a practically good solution. We propose a branch-and-cut algorithm to solve our model. To our knowledge, this novel methodology has not been explored to solve MVPDPTW problem.

We start by relaxing our DFJ constraints. Let Formulation 1 with DFJ constraints relaxation be our Master Relaxation Problem (MRP). Our algorithm begins by solving the MRP in a Branch-and-Bound (BB) framework. Each node of this $\mathrm{BB}$ tree can represent one of the following cases:

1. Feasible routes form origin to destination depots for all vehicles, in which case the BB node is pruned by feasibility and the incumbent solution is updated as required.

2. A fractional solution, in which case we continue branching.

3. An infeasible LP relaxation, in which case we prune that $\mathrm{BB}$ node by infeasibility.

4. Vehicle routes with disjoint sub-tours feasible to the MRP, then a sequence of steps is followed as shown below:

a. The separation problem is solved and a sub-tour for one vehicle is detected.

b. The DFJ constraint is added to the current formulation as a lazy constraint and the LP relaxation is re-solved.

c. The re-solved LP may terminate yielding feasible routes for all vehicles (in which case the BB node is pruned by feasibility) or a fractional solution (in which case we continue branching) or vehicle routes with disjoint subtours (in which case steps a-c are repeated).

This algorithm terminates when there are no nodes left in the BB tree to branch. At that point, the incumbent solution is feasible routes from origin to destination for all vehicles.

\subsection{Implementation statement}

Real-world test instances from a Logistics company were used for testing our model results. Ten instances transported by the company using Less-than truckload (LTL) mode on May 2016 were tested. Each instance has 10 shipment orders, 22 vertices (complete graph for pickup and delivery) and time windows for every pickup and delivery node. Maximum time a driver can spend on a road were fixed to be 55 hours (excluding breaks).

The branch-and-cut algorithm for our model was implemented using $\mathrm{C}++$ and Gurobi ${ }^{\mathrm{TM}}$ 7.5.2 on dual Intel $^{\circledR}$ Xeon E5-.2620 Sandy Bridge hex core $2.0 \mathrm{GHz}$ CPU, with 32 GB RAM.

For the remainder of this paper, we will call the actual routes used by the company as "unconsolidated" and routes identified by our model as "consolidated".

\section{Environmental impact measurement}

In this section, we present our emission quantification method. Our aim in emission studies is to quantify and compare the emission rates from unconsolidated routes against the consolidated routes from our model. We focus on three major pollutants from trucks, namely Carbon dioxide $\left(\mathrm{CO}_{2}\right)$, Oxides of Nitrogen (NOx), and Particulate Matter (PM).

To measure emission levels, we calculate $\mathrm{kg}$ of greenhouse gas emission for unconsolidated and consolidated routes. For this calculation, we use emission rates per ton of cargo as presented by Carbonfund [13], Environment Protection Agency (EPA) [14] and Agar et al. [15]. We use emission rates under Heavy Duty Diesel Vehicles (HDDV) category. Our emission rate calculations are based on the estimates from EPA. We refer the reader to the handbook on Federal Highway Administration (FHWA) emission rates [16]. The emission rates from the FHWA handbook and our source are very close. For example, FHWA refers to $10.15 \mathrm{~kg}$ of $\mathrm{CO}_{2}$ emission for 1 gallon of diesel (From 2013), whereas EPA refers to $10.21 \mathrm{~kg}$ of $\mathrm{CO}_{2}$ emission for 1 gallon of diesel (From 2015). Since there is no huge disparity in the emission rates between the two sources and since EPA has the latest numbers, we did not do a recalibration according to FHWA data.

Even after using the consolidated routes, it is noteworthy that the ton-mile cargo transportation does not change very drastically. For example, if 1-ton shipment is to be transported from Chicago to Dallas, then that total ton-miles is not going to change very much between unconsolidated and consolidated scenario. So, it follows that we may not find a huge emission level difference with cargo weight. However, we do identify 
routes using fewer number of trucks (from results and discussion section). Let us assume that an empty truck with trailer weighs around $10,887 \mathrm{~kg}$ [17]. By removing a single empty truck from the road, we remove 10,887 $\mathrm{kg}$ of emission source from the road. We present benefits resulting from this empty truck weight reduction.

We use monetary equivalent to compare emission level between our solution and unconsolidated routes. Hwang [5] used a monetary equivalent loss value for different types of greenhouse gases. Using this conversion factor, we can compute the equivalent loss value for emission levels. The cost conversion factor for different gases are shown in Table 2.

Table 2. Emission rates and monetary loss equivalent for various emission gases.

\begin{tabular}{|c|c|c|c|c|c|}
\hline \multicolumn{2}{|c|}{ Emission rate (kg/tonne-mile) } & \multicolumn{3}{|c|}{$\begin{array}{c}\text { Monetary loss } \\
\text { equivalent (\$/kg) }\end{array}$} \\
\hline $\mathrm{CO}_{2}$ & $\mathrm{NO}_{\mathrm{x}}$ & $\mathrm{PM}$ & $\mathrm{CO}_{2}$ & $\mathrm{NO}_{\mathrm{x}}$ & $\mathrm{PM}$ \\
\hline 0.14645 & $9.80 \mathrm{E}-04$ & $4.67 \mathrm{E}-05$ & 0.28 & 0.2 & 0.3 \\
\hline
\end{tabular}

\section{Results and discussion}

\subsection{Closer look at a single instance}

We begin this section by focusing on the results from a single instance: the unconsolidated and consolidated routes for that network. In unconsolidated scenario, 10 trucks were used covering a total of 7533.75 miles and with total operation cost of $\$ 13,116.49$, whereas consolidated routes delivered same shipments with 5 trucks covering 4355.89 miles and total cost of $\$ 7369.63$. Figure 1 shows the enhanced comparison between unconsolidated and consolidated routes along the east coast. From that figure, we can see that our model routes are more cost effective than the unconsolidated routes.

\subsection{Results from all instances}

We compared consolidated routes from our model with actual routes used by the company on 10 different instances. Each instance has 10 shipment orders from single origin to a single destination. The results are shown in Table 3. The unconsolidated fields are actual routes. Whereas, the consolidated fields show the results attained by our model. It is noteworthy, that the solution from our model still respects customer time window constraints, vehicle capacity constraints and maximum time on a road for a truck driver.

From the results, we can see that the trucks used in the consolidated case is fewer than in unconsolidated routes. For all the 10 instances, our model found consolidated routes are less expensive than unconsolidated routes.

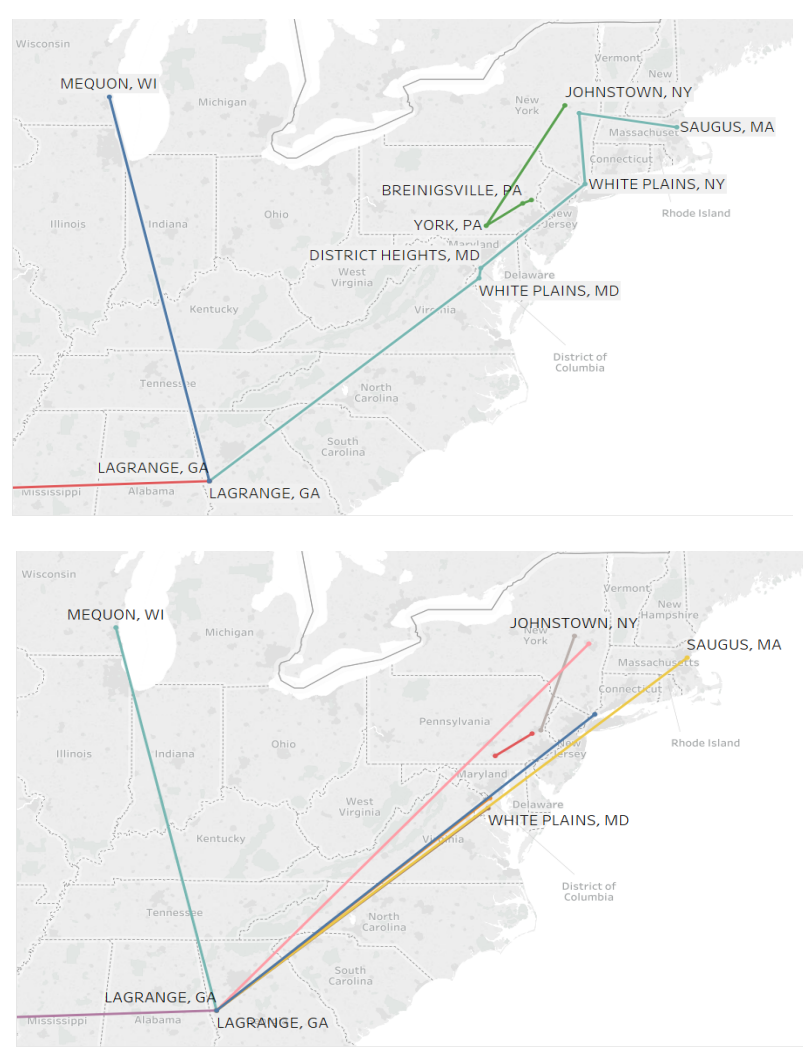

Fig. 1. Unconsolidated routes (top) and consolidated routes (bottom) along east coast.

Table 3. Comparison between unconsolidated and consolidated routes on different instances.

\begin{tabular}{|c|c|c|c|c|c|c|c|c|}
\hline \multirow{2}{*}{$\begin{array}{c}\text { Network } \\
\text { ID }\end{array}$} & \multicolumn{2}{|c|}{ No of Trucks } & \multicolumn{2}{c|}{ Total cost (\$) } & \multicolumn{2}{c|}{ Total Distance (miles) } & \multicolumn{2}{c|}{ Emission loss (\$) } \\
\cline { 2 - 9 } & Unconsolidated & Consolidated & Unconsolidated & Consolidated & Unconsolidated & Consolidated & Unconsolidated & Consolidated \\
\hline 1 & 10 & 5 & $\$ 13,116$ & $\$ 7,369$ & 7,534 & 4,356 & $\$ 3,381$ & $\$ 1,955$ \\
\hline 2 & 10 & 5 & $\$ 7,577$ & $\$ 6,018$ & 3,519 & 3,375 & $\$ 1,579$ & $\$ 1,515$ \\
\hline 3 & 10 & 6 & $\$ 9,107$ & $\$ 6,618$ & 4,628 & 3,613 & $\$ 2,077$ & $\$ 1,621$ \\
\hline 4 & 10 & 7 & $\$ 6,483$ & $\$ 5,627$ & 2,727 & 2,698 & $\$ 1,224$ & $\$ 1,211$ \\
\hline 5 & 10 & 8 & $\$ 5,621$ & $\$ 4,669$ & 2,102 & 1,807 & $\$ 943$ & $\$ 811$ \\
\hline 6 & 10 & 5 & $\$ 3,414$ & $\$ 1,918$ & 503 & 404 & $\$ 226$ & $\$ 181$ \\
\hline 7 & 10 & 8 & $\$ 15,466$ & $\$ 13,096$ & 9,236 & 7,913 & $\$ 4,144$ & $\$ 3,551$ \\
\hline 8 & 10 & 7 & $\$ 10,617$ & $\$ 9,635$ & 5,723 & 5,602 & $\$ 2,568$ & $\$ 2,514$ \\
\hline 9 & 10 & 6 & $\$ 6,021$ & $\$ 4,664$ & 2,392 & 2,197 & $\$ 1,073$ & $\$ 986$ \\
\hline 10 & 10 & 6 & $\$ 5,669$ & $\$ 3,996$ & 2,137 & 1,691 & $\$ 959$ & $\$ 759$ \\
\hline
\end{tabular}


The total cost in our results include the fixed cost for dispatching a truck and the traveling cost based on traveling distance. The fixed cost for dispatching a truck includes trailer rent, licensing, fixed office cost, and cargo, collision, bobtail and life insurance. Using the estimates from [18], we assume the operating cost for trucks to be $\$ 1.38$ per mile. Using the estimates from [19], we also assume that the fixed cost of dispatching a truck is $\$ 272$ per day. This total cost is the objective function that we seek to minimize in our MIP model. The distances covered in both cases are also presented in Table 3. Our model identified routes which are shorter in comparison to unconsolidated routes.

We can also see that the quantified emission loss is lower from the results of our model. So, our model routes are more environment friendly than the unconsolidated case. A case-by-case comparison of emission levels is shown in Figure 2.

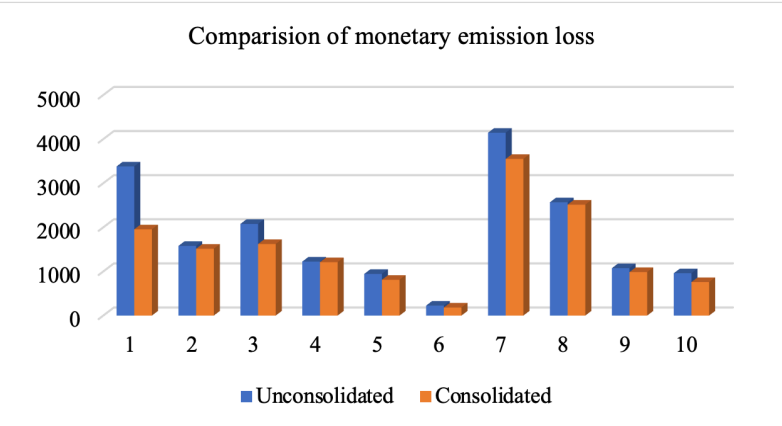

Fig. 2. Emission level comparison on all instances (unconsolidated vs consolidated).

\subsection{Computational results}

We show the computational results of our model and algorithm in Table 4. For these real-world medium size instances, the runtimes of our algorithm are fairly short. The number of Branch-and-Bound (BB) nodes and time taken for solving root node Linear Program (LP) relaxation are also shown in the results. From the number of $\mathrm{BB}$ nodes, we can see that our LP relaxation is not very strong. With a tighter LP relaxation, we can solve larger instances quickly. As is, the growth of our model (\# of variables and constraints) is exponential with respect to \# of shipments.

Table 4. Computational results for our MVPDPTW model and algorithm.

\begin{tabular}{|c|c|c|c|}
\hline Instance & $\begin{array}{c}\text { Runtime } \\
\text { (seconds) }\end{array}$ & $\begin{array}{c}\text { \#B\&B } \\
\text { nodes }\end{array}$ & $\begin{array}{c}\text { Root node solving } \\
\text { time (seconds) }\end{array}$ \\
\hline 1 & 279.97 & $1,70,422$ & 4.09 \\
\hline 2 & 108.72 & 3,063 & 1.43 \\
\hline 3 & 89.38 & 419 & 1.46 \\
\hline 4 & 240.65 & 1,848 & 1.39 \\
\hline 5 & 5.75 & 228 & 0.62 \\
\hline 6 & 77.43 & 74,369 & 2.87 \\
\hline 7 & 183.09 & 3,787 & 2.48 \\
\hline 8 & 281.63 & 7,886 & 1.80 \\
\hline 9 & 104.85 & 1 & 1.05 \\
\hline 10 & 375.80 & 24,939 & 2.57 \\
\hline
\end{tabular}

\section{Conclusions and future works}

In this paper, we presented a pickup and delivery model to identify effective cargo consolidation strategies. Our model was tested on multiple real-world freight movement instances. We considered constraints like maximum on-road time for truck driver, truck capacity and delivery time window constraints. The test results verify that the consolidation strategies identified by our model are more cost effective for logistics companies.

We used a quantification technique to compare the emission rates between actual routes and our model routes. Certain pollutants were converted into monetary loss values. From the emission results, we showed that our model routes are more environment friendly (lower emission loss values) than actual used routes.

\section{References}

1. Parragh, S.N., Doerner, K.F., and Hartl, R.F. (2008). A survey on pickup and delivery problems, Journal fur Betriebswirtschaft, 21-51.

2. Hoff, A., Anderson, H., Christiansen, M., Hasle G., and Løkketangen, A. (2010). Industrial aspects and literature survey: Fleet composition and routing, Computers \& Operations Research, 37(9), 20412061.

3. Ropke, S., and Cordeau, J.-F. (2009). Branch and cut and price for the pickup and delivery problem with time windows. Transportation Science, 267286.

4. Schulte, F., Lalla-Ruiz, E., González-Ramírez, R.G., and Voß, S. (2017). Reducing port-related empty truck emissions: A mathematical approach for truck appointments with collaboration, Transportation Research Part E.

5. Hwang, T.S. (2014). Freight demand modeling and logistics planning for assessment of freight systems' environmental impacts, University of Illinois at Urbana-Champaign, Chicago.

6. Tarantilis, C., and Kiranoudis, C. (2007). A flexible adaptive memory-based algorithm for real-life transportation operations: Two case studies from dairy and construction sector, European Journal of Operational Research, 179(3), 806-822.

7. Tavakkoli-Moghaddam, R., Safaei, N., Kah, M., and Rabbani, M. (2007). A new capacitated vehicle routing problem with split service for minimizing fleet cost by simulated annealing, Journal of the Franklin Institute, 344(5), 406-425.

8. Bettinelli, A., Ceselli, A., and Righini, G. (2011). A branch-and-cut-and-price algorithm for the multidepot heterogeneous vehicle routing problem with time windows, Transportation Research Part C: Emerging Technologies, 723-740.

9. Haghani, A., and Oh, S.C. (1996). Formulation and solution of a multi-commodity, multi-modal network flow model for disaster relief operations, Transportation Research Part A: Policy and Practice, 231-250. 
10. California Department of Transportation. (2016). Trucking industry overview. [Online]. Available: http://www.dot.ca.gov/hq/tpp/offices/ogm/key reports files/National,\%20Technical\%20studies/Trucking industry overview.pdf.

11. American Trucking Association. (2017). Reports, trends \& statistics. [Online]. Available: http://www.trucking.org/News_and Information Reports Industry Data.aspx.

12. Environmental Defense Fund. (2016). Green freight facts \& figures. [Online]. Available: http://business.edf.org/projects/greenfreight-freightfacts-figures.

13. Carbonfund. (2015). How we calculate. [Online]. Available: http://carbonfund.org/how-we-calculate/.

14. Environment Protection Agency. (2015). Emission factors for greenhouse gas emission. [Online]. Available:

https://www.epa.gov/sites/production/files/201512/documents/emission-factors nov 2015.pdf.

15. Agar, B.J., Baetz, B.W., and Wilson, B.G. (2007). "Fuel consumption, emissions estimation, and emissions cost estimates using global positioning data, Journal of the Air \& Waste Management Association, 348-354.

16. Federal Highway Administration. (2013). Handbook for Estimating Transportation Greenhouse Gases for Integration into the Planning Process, Federal Highway Administration, [Online]. Available: https://www.fhwa.dot.gov/environment/sustainabilit y/energy/publications/ghg handbook/ghghandbook. pdf.

17. Department of Energy. (2010). Fact \#621: May 3, 2010 Gross Vehicle Weight vs. Empty Vehicle Weight, [Online]. Available:https://www.energy.gov/eere/vehicles/fact -621-may-3-2010-gross-vehicle-weight-vs-emptyvehicle-weight.

18. The Truckers Report. (2013). The Real Cost of Trucking - Per Mile Operating Cost of a Commercial Truck. [Online]. Available: https://www.thetruckersreport.com/infographics/cos t-of-trucking/.

19. Transportation Business Associates. (2010). Fixed Expenses. [Online]. Available: http://www.tbabz.com/wp/tractor-trailer-ops/fixedexpenses. 